\title{
Spontaneous Emission by Three-level Atoms Pumped by Electron Bombardment
}

\author{
Menisha Alemu \\ Department of Physics, Wolkite University, P. O. Box 07, Wolkite, Ethiopia
}

Received October 14, 2019; Revised November 25, 2019; Accepted December 30, 2019

Copyright $\odot 2020$ by authors, all rights reserved. Authors agree that this article remains permanently open access under the terms of the Creative Commons Attribution License 4.0 International License

\begin{abstract}
In this paper we have studied the statistical and squeezing properties of the cavity light generated by a threelevel laser. In this quantum optical system, $\mathrm{N}$ three-level atoms available in an open cavity, coupled to a two-mode vacuum reservoir, are pumped to the top level by means of electron bombardment at constant rate. We have considered the case in which the three-level atoms and the cavity modes interact with the two-mode vacuum reservoir. We have carried out our analysis by putting the noise operators associated with the vacuum reservoir in normal order. Applying the solutions of the equations of evolution for the expectation values of the atomic operators and the quantum Langevin equations for the cavity mode operators, we have calculated the mean and variance of the photon number as well as the quadrature squeezing for the cavity light. In addition, we have shown that the presence of the spontaneous emission process leads to a decrease in the mean and variance of the photon number. We have observed that the two-mode cavity light is in a squeezed state and the squeezing occurs in the minus quadrature. The maximum quadrature squeezing of the light generated by the laser, operating far below threshold, is found to be $50 \%$ below the vacuum-state level. We have also established that the mean photon number in the presence of spontaneous emission is less than the absence of spontaneous emission.
\end{abstract}

Keywords Operator Dynamics, Photon Statistics, Quadrature Squeezing, Spontaneous Emission

\section{Introduction}

A three-level laser is a quantum optical system in which light is generated by three-level atoms inside a cavity usually coupled to a vacuum reservoir. In one mode of such a laser, three-level atoms initially prepared in a coherent superposition of the top and bottom levels are injected into a cavity and then removed after they decayed due to spontaneous emission $[1,2,3,4]$. In another model, the top and bottom levels of the three-level atoms injected into a cavity are coupled by coherent light $[5,6]$. The statistical and squeezing properties of the light generated by three-level lasers have been investigated by several authors $[7,8,9,10,11,12]$. It is found that a three-level laser in either model generates squeezed light under certain conditions. It appears to be quit difficult to prepare the atom in a coherent superposition of the top and bottom levels before they are injected into the laser cavity. In addition, it should certainly be hard to find out that the atoms have decayed spontaneously before they are removed from the cavity.

Moreover, Fesseha $[9,10]$ has studied the squeezing and the statistical properties of the light produced by a three-level laser with the atoms in a closed cavity and pumped by electron bombardment. He has shown that the maximum quadrature squeezing of the light generated by the laser, operating below threshold, is found to be $50 \%$ below the vacuum-state level. In addition, he has also found that the quadrature squeezing of the output light is equal to that of the cavity light. On the other hand, this study shows that the local quadrature squeezing is greater than the global quadrature squeezing. He has also found that a large part of the total mean photon number is confined in a relatively small frequency interval. Furthermore, has studied the squeezing and the statistical properties of the light produced by a three-level laser with the atoms in a closed cavity and pumped by coherent light. He has shown that the maximum quadrature squeezing is $43 \%$ below the vacuum-state level, which is slightly less than the result found with electron bombardment.

In this paper, we seek to analyze the quantum properties of light emitted by three-level atoms available in an open cavity and pumped to the top level by electron bombardment. Thus taking into account the interaction of three-level atoms 


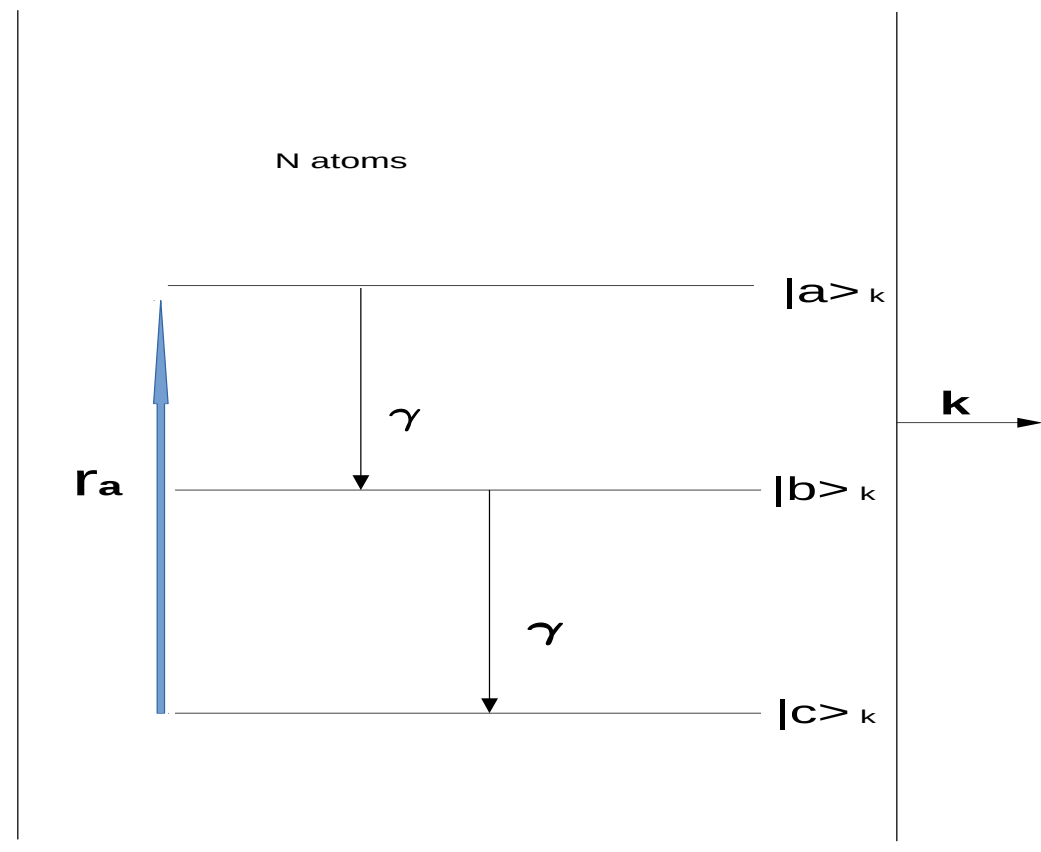

Figure 1. Schematic representation of a three-level laser coupled to a two-mode vacuum reservoir.

with a resonant cavity light and the damping of the cavity light by a vacuum reservoir, we obtain the photon statistics, the quadrature variance, and the quadrature squeezing of the cavity light. We carry out our calculation by putting the noise operators associated with the vacuum reservoir in normal order and considering the interaction of the three-level atoms with the vacuum reservoir out side the cavity light.

\section{Operator dynamics}

We consider here the case in which $\mathrm{N}$ three-level atoms in a cascade configuration and available in an open cavity. We denote the top, intermediate, and bottom levels of these atoms by $|a\rangle_{k},|b\rangle_{k}$, and $|c\rangle_{k}$, respectively. We prefer to call the light emitted from the top level light mode $a$ and the one emitted from the intermediate level light mode $b$. We carry out our analysis with light modes $a$ and $b$ having the same or different frequencies. In addition, we assume that light modes $a$ and $b$ to be at resonance with the two transitions $|a\rangle_{k} \rightarrow|b\rangle_{k}$ and $|b\rangle_{k} \rightarrow|c\rangle_{k}$, with direct transition between $|a\rangle_{k}$ and $|c\rangle_{k}$ to be electric-dipole forbidden. The interaction of a three-level atoms with cavity modes $a$ and $b$ can be described at resonance by the Hamiltonian

$$
\hat{H}=i g\left(\hat{\sigma}_{a}^{\dagger k} \hat{a}-\hat{a}^{\dagger} \hat{\sigma}_{a}^{k}+\hat{\sigma}_{b}^{\dagger k} \hat{b}-\hat{b}^{\dagger} \hat{\sigma}_{b}^{k}\right),
$$

where

$$
\hat{\sigma}_{a}^{k}=|b\rangle_{k k}\langle a|
$$

and

$$
\hat{\sigma}_{b}^{k}=|c\rangle_{k k}\langle b|
$$

are lowering atomic operators, $\hat{a}$ and $\hat{b}$ are the annihilation operators for the cavity modes, $\mathrm{g}$ is the coupling constant between the atom and the cavity modes. We assume that the cavity modes are coupled to a two-mode vacuum reservoir via a singleport mirror. In addition, we carry out our calculation by putting the noise operators associated with the vacuum reservoir in normal order. Thus the noise operators will not have any effect on the dynamics of the cavity mode operators $[9,10]$. We can therefore drop the noise operator and write the quantum Langevin equation for the operators $\hat{a}$ and $\hat{b}$ by

$$
\begin{aligned}
\frac{d \hat{a}}{d t} & =-\frac{\kappa}{2} \hat{a}-i[\hat{a}, \hat{H}], \\
\frac{d \hat{b}}{d t} & =-\frac{\kappa}{2} \hat{b}-i[\hat{b}, \hat{H}],
\end{aligned}
$$


where $\kappa$ is the cavity damping constant. With the aid of Eqs. (1), (4), and (5), one can easily establish that

$$
\begin{aligned}
& \frac{d \hat{a}}{d t}=-\frac{\kappa}{2} \hat{a}-g \hat{\sigma}_{a}^{k}, \\
& \frac{d \hat{b}}{d t}=-\frac{\kappa}{2} \hat{b}-g \hat{\sigma}_{b}^{k} .
\end{aligned}
$$

Furthermore, the master equation for a three-level atom interacting with a vacuum reservoir is given by [10]

$$
\begin{aligned}
\frac{d \hat{\rho}}{d t}= & -i[\hat{H}, \hat{\rho}]+\frac{\gamma}{2}\left[2 \hat{\sigma}_{a}^{k} \hat{\rho} \hat{\sigma}_{a}^{\dagger k}-\hat{\sigma}_{a}^{\dagger k} \hat{\sigma}_{a}^{k} \hat{\rho}-\hat{\rho} \hat{\sigma}_{a}^{\dagger k} \hat{\sigma}_{a}^{k}\right] \\
& +\frac{\gamma}{2}\left[2 \hat{\sigma}_{b}^{k} \hat{\rho} \hat{\sigma}_{b}^{\dagger k}-\hat{\sigma}_{b}^{\dagger k} \hat{\sigma}_{b}^{k} \hat{\rho}-\hat{\rho} \hat{\sigma}_{b}^{\dagger k} \hat{\sigma}_{b}^{k}\right],
\end{aligned}
$$

where $\gamma$ is the spontaneous emission decay constant. We can rewrite Eq. (8) as

$$
\frac{d \hat{\rho}}{d t}=-i[\hat{H}, \hat{\rho}]+\frac{\gamma}{2}\left[2 \hat{\sigma}_{a}^{k} \hat{\rho} \hat{\sigma}_{a}^{\dagger k}-\hat{\eta}_{a}^{k} \hat{\rho}-\hat{\rho} \hat{\eta}_{a}^{k}+2 \hat{\sigma}_{b}^{k} \hat{\rho} \hat{\sigma}_{b}^{\dagger k}-\hat{\eta}_{b}^{k} \hat{\rho}-\hat{\rho} \hat{\eta}_{b}^{k}\right],
$$

where

$$
\begin{aligned}
& \hat{\eta}_{a}^{k}=|a\rangle_{k k}\langle a|, \\
& \hat{\eta}_{b}^{k}=|b\rangle_{k k}\langle b| .
\end{aligned}
$$

Using Eq. (1), we can put Eq. (9) in the form

$$
\begin{aligned}
\frac{d \hat{\rho}}{d t} & =g\left[\hat{\sigma}_{a}^{\dagger k} \hat{a} \hat{\rho}-\hat{\rho} \hat{\sigma}_{a}^{\dagger k} \hat{a}+\hat{\sigma}_{b}^{\dagger k} \hat{b} \hat{\rho}-\hat{\rho} \hat{\sigma}_{b}^{\dagger k} \hat{b}-\hat{a}^{\dagger} \hat{\sigma}_{b}^{k} \hat{\rho}-\hat{b}^{\dagger} \hat{\sigma}_{b}^{k} \hat{\rho}+\hat{\rho} \hat{a}^{\dagger} \hat{\sigma}_{a}^{k}+g \hat{\rho} \hat{b}^{\dagger} \hat{\sigma}_{b}^{k}\right] \\
& +\frac{\gamma}{2}\left[2 \hat{\sigma}_{a}^{k} \hat{\rho} \hat{\sigma}_{b}^{\dagger k}-\hat{\eta}_{a}^{k} \hat{\rho}-\hat{\rho} \hat{\eta}_{a}^{k}+2 \hat{\sigma}_{b}^{k} \hat{\rho} \hat{\sigma}_{b}^{\dagger k}-\hat{\eta}_{b}^{k} \hat{\rho}-\hat{\rho} \hat{\eta}_{b}^{k}\right] .
\end{aligned}
$$

Now applying the relation

$$
\frac{d}{d t}\langle\hat{A}\rangle=\operatorname{Tr}\left(\frac{d \rho}{d t} \hat{A}\right)
$$

along with Eq. (12), we can easily establish that

$$
\begin{gathered}
\frac{d}{d t}\left\langle\hat{\sigma}_{a}^{k}\right\rangle=-\gamma\left\langle\hat{\sigma}_{a}^{k}\right\rangle+g\left[\left\langle\hat{\eta}_{b}^{k} \hat{a}\right\rangle-\left\langle\hat{\eta}_{a}^{k} \hat{a}\right\rangle+\left\langle\hat{b}^{\dagger} \hat{\sigma}_{c}^{k}\right\rangle\right] \\
\frac{d}{d t}\left\langle\hat{\sigma}_{b}^{k}\right\rangle=-\frac{\gamma}{2}\left\langle\hat{\sigma}_{b}^{k}\right\rangle+g\left[\left\langle\hat{\eta}_{c}^{k} \hat{b}\right\rangle-\left\langle\hat{\eta}_{b}^{k} \hat{b}\right\rangle+\left\langle\hat{a}^{\dagger} \hat{\sigma}_{c}^{k}\right\rangle\right] \\
\frac{d}{d t}\left\langle\hat{\sigma}_{c}^{k}\right\rangle=-\frac{\gamma}{2}\left\langle\hat{\sigma}_{c}^{k}\right\rangle+g\left[\left\langle\hat{\sigma}_{b}^{k} \hat{a}\right\rangle-\left\langle\hat{\sigma}_{a}^{k} \hat{b}\right\rangle\right] \\
\frac{d}{d t}\left\langle\hat{\eta}_{a}^{k}\right\rangle=-\gamma\left\langle\hat{\eta}_{a}^{k}\right\rangle+g\left[\left\langle\hat{\sigma}_{a}^{\dagger k} \hat{a}\right\rangle+\left\langle\hat{a}^{\dagger} \hat{\sigma}_{a}^{k}\right\rangle\right] \\
\frac{d}{d t}\left\langle\hat{\eta}_{b}^{k}\right\rangle=\gamma\left[\left\langle\hat{\eta}_{a}^{k}\right\rangle-\left\langle\hat{\eta}_{b}^{k}\right\rangle\right]+g\left[\left\langle\hat{b}^{\dagger} \hat{\sigma}_{b}^{k}\right\rangle+\left\langle\hat{\sigma}_{b}^{\dagger k} \hat{b}\right\rangle-\left\langle\hat{\sigma}_{a}^{\dagger k} \hat{a}\right\rangle-\left\langle\hat{a}^{\dagger} \hat{\sigma}_{a}^{k}\right\rangle\right] \\
\frac{d}{d t}\left\langle\hat{\eta}_{c}^{k}\right\rangle=\gamma\left\langle\hat{\eta}_{b}^{k}\right\rangle-g\left[\left\langle\hat{b}^{\dagger} \hat{\sigma}_{b}^{k}\right\rangle+\left\langle\hat{\sigma}_{b}^{\dagger k} \hat{b}\right\rangle\right]
\end{gathered}
$$

where

$$
\hat{\sigma}_{c}^{k}=|c\rangle_{k k}\langle a|
$$

and

$$
\hat{\eta}_{c}^{k}=|c\rangle_{k k}\langle c| .
$$

We see that Eqs. (14)-(19) are nonlinear differential equations and hence it is not possible to find exact time-dependent solutions of these equations. We intend to over come this problem by applying the large-time approximation [13]. Then using this approximation scheme, we get from Eqs. (6) and (7) the approximately valid relations

$$
\hat{a}=-\frac{2 g}{\kappa} \hat{\sigma}_{a}^{k}
$$




$$
\hat{b}=-\frac{2 g}{\kappa} \hat{\sigma}_{b}^{k} .
$$

Evidently, these would turn out to be exact relations at steady state. Now combining Eqs. (22) and (23) with Eqs. (14)-(19), we get

$$
\begin{aligned}
\frac{d}{d t}\left\langle\hat{\sigma}_{a}^{k}\right\rangle & =-\left[\gamma+\gamma_{c}\right]\left\langle\hat{\sigma}_{a}^{k}\right\rangle, \\
\frac{d}{d t}\left\langle\hat{\sigma}_{b}^{k}\right\rangle & =-\left[\frac{\gamma}{2}+\frac{\gamma_{c}}{2}\right]\left\langle\hat{\sigma}_{b}^{k}\right\rangle, \\
\frac{d}{d t}\left\langle\hat{\sigma}_{c}^{k}\right\rangle & =-\left[\frac{\gamma}{2}+\frac{\gamma_{c}}{2}\right]\left\langle\hat{\sigma}_{c}^{k}\right\rangle, \\
\frac{d}{d t}\left\langle\hat{\eta}_{a}^{k}\right\rangle & =-\left[\gamma+\gamma_{c}\right]\left\langle\hat{\eta}_{a}^{k}\right\rangle, \\
\frac{d}{d t}\left\langle\hat{\eta}_{b}^{k}\right\rangle=-\left[\gamma+\gamma_{c}\right]\left\langle\hat{\eta}_{b}^{k}\right\rangle+\left[\gamma+\gamma_{c}\right]\left\langle\hat{\eta}_{a}^{k}\right\rangle & \\
\frac{d}{d t}\left\langle\hat{\eta}_{c}^{k}\right\rangle & =\left[\gamma+\gamma_{c}\right]\left\langle\hat{\eta}_{b}^{k}\right\rangle,
\end{aligned}
$$

where

$$
\gamma_{c}=\frac{4 g^{2}}{\kappa}
$$

is the stimulated emission decay constant. We note that Eqs. (24) - (29) represent the equation of evolution for the atomic operators in the absence of the pumping process. We thus want to include the pumping process. The pumping process must surely affect the dynamics of $\left\langle\hat{\eta}_{a}^{k}\right\rangle$ and $\left\langle\hat{\eta}_{c}^{k}\right\rangle$. We seek here to pump the atoms by electron bombardment. If $r_{a}$ represents the rate at which a single atom is pumped from the bottom to the top level, then $\left\langle\hat{\eta}_{a}^{k}\right\rangle$ increases at the rate of $r_{a}\left\langle\hat{\eta}_{c}^{k}\right\rangle$ and $\left\langle\hat{\eta}_{c}^{k}\right\rangle$ decreases at the same rate. In view of this, we rewrite Eqs. (27) and (29) as

$$
\begin{aligned}
\frac{d}{d t}\left\langle\hat{\eta}_{a}^{k}\right\rangle & =-\left[\gamma+\gamma_{c}\right]\left\langle\hat{\eta}_{a}^{k}\right\rangle+r_{a}\left\langle\hat{\eta}_{c}^{k}\right\rangle, \\
\frac{d}{d t}\left\langle\hat{\eta}_{c}^{k}\right\rangle & =\left[\gamma+\gamma_{c}\right]\left\langle\hat{\eta}_{b}^{k}\right\rangle-r_{a}\left\langle\hat{\eta}_{c}\right\rangle .
\end{aligned}
$$

We next sum Eqs. (24), (25), (28), (31), and (32) over the $\mathrm{N}$ three-level atoms, so that

$$
\begin{gathered}
\frac{d}{d t}\left\langle\hat{m}_{a}\right\rangle=-\left[\gamma+\gamma_{c}\right]\left\langle\hat{m}_{a}\right\rangle, \\
\frac{d}{d t}\left\langle\hat{m}_{b}\right\rangle=-\left[\frac{\gamma}{2}+\frac{\gamma_{c}}{2}\right]\left\langle\hat{m}_{b}\right\rangle, \\
\frac{d}{d t}\left\langle\hat{N}_{a}\right\rangle=-\left[\gamma+\gamma_{c}\right]\left\langle\hat{N}_{a}\right\rangle+r_{a}\left\langle\hat{N}_{c}\right\rangle, \\
\frac{d}{d t}\left\langle\hat{N}_{b}\right\rangle=-\left[\gamma+\gamma_{c}\right]\left\langle\hat{N}_{b}\right\rangle+\left[\gamma+\gamma_{c}\right]\left\langle\hat{N}_{a}\right\rangle, \\
\frac{d}{d t}\left\langle\hat{N}_{c}\right\rangle=\left[\gamma+\gamma_{c}\right]\left\langle\hat{N}_{b}\right\rangle-r_{a}\left\langle\hat{N}_{c}\right\rangle,
\end{gathered}
$$

in which

$$
\begin{aligned}
& \hat{m}_{a}=\sum_{k=1}^{N} \hat{\sigma}_{a}^{k}, \\
& \hat{m}_{b}=\sum_{k=1}^{N} \hat{\sigma}_{b}^{k}, \\
& \hat{N}_{a}=\sum_{k=1}^{N} \hat{\eta}_{a}^{k}, \\
& \hat{N}_{b}=\sum_{k=1}^{N} \hat{\eta}_{b}^{k},
\end{aligned}
$$




$$
\hat{N}_{c}=\sum_{k=1}^{N} \hat{\eta}_{c}^{k}
$$

with the operators $\hat{N}_{a}, \hat{N}_{b}$, and $\hat{N}_{c}$ representing the number of atoms in the top, intermediate, and bottom levels. In addition, employing the completeness relation

$$
\hat{\eta}_{a}^{k}+\hat{\eta}_{b}^{k}+\hat{\eta}_{c}^{k}=\hat{I}
$$

we easily arrive at

$$
\left\langle\hat{N}_{a}\right\rangle+\left\langle\hat{N}_{b}\right\rangle+\left\langle\hat{N}_{c}\right\rangle=N
$$

Furthermore, applying the definition given by Eq. (2) and setting for any k

$$
\hat{\sigma}_{a}^{k}=|b\rangle\langle a|,
$$

we have

$$
\hat{m}_{a}=N|b\rangle\langle a| \text {. }
$$

Following the same procedure, one can also check that

$$
\begin{gathered}
\hat{m}_{b}=N|c\rangle\langle b|, \\
\hat{m}_{c}=N|c\rangle\langle a|, \\
\hat{N}_{a}=N|a\rangle\langle a|, \\
\hat{N}_{b}=N|b\rangle\langle b|, \\
\hat{N}_{c}=N|c\rangle\langle c|,
\end{gathered}
$$

where

$$
\hat{m}_{c}=\sum_{k=1}^{N} \hat{\sigma}_{c}^{k}
$$

Moreover, using the definition

$$
\hat{m}=\hat{m}_{a}+\hat{m}_{b}
$$

and taking into account Eqs. (46)-(51), it can be readily established that

$$
\begin{gathered}
\hat{m}^{\dagger} \hat{m}=N\left(\hat{N}_{a}+\hat{N}_{b}\right), \\
\hat{m} \hat{m}^{\dagger}=N\left(\hat{N}_{b}+\hat{N}_{c}\right), \\
\hat{m}^{2}=N \hat{m}_{c} .
\end{gathered}
$$

With the aid of Eq. (44), one can put Eq. (35) in the form

$$
\frac{d}{d t}\left\langle\hat{N}_{a}\right\rangle=-\left[\gamma+\gamma_{c}+r_{a}\right]\left\langle\hat{N}_{a}\right\rangle+r_{a}\left[N-\left\langle\hat{N}_{b}\right\rangle\right]
$$

Applying the large-time approximation scheme to Eq. (36), we get

$$
\left\langle\hat{N}_{b}\right\rangle=\left\langle\hat{N}_{a}\right\rangle
$$

Thus on taking into account this result, Eq. (57) can be written as

$$
\frac{d}{d t}\left\langle\hat{N}_{a}\right\rangle=-\left[\gamma+\gamma_{c}+2 r_{a}\right]\left\langle\hat{N}_{a}\right\rangle+N r_{a} .
$$

The steady-state solution of Eq. (59) is expressible as

$$
\left\langle\hat{N}_{a}\right\rangle=\frac{r_{a} N}{\gamma+\gamma_{c}+2 r_{a}} .
$$

Using the steady-state solution of Eq. (37) along with Eq. (58), we have

$$
\left\langle\hat{N}_{c}\right\rangle=\frac{\gamma+\gamma_{c}}{r_{a}}\left\langle\hat{N}_{a}\right\rangle
$$


On account of Eq. (60), Eq. (61) takes the form

$$
\left\langle\hat{N}_{c}\right\rangle=\frac{\left(\gamma+\gamma_{c}\right) N}{\gamma+\gamma_{c}+2 r_{a}} .
$$

For $r_{a}=0$, we see that $\left\langle\hat{N}_{a}\right\rangle=\left\langle\hat{N}_{b}\right\rangle=0$ and $\left\langle\hat{N}_{c}\right\rangle=N$. This result holds whether the atoms are initially in the top or bottom level.

In the presence of $\mathrm{N}$ three-level atoms, we rewrite Eq. (6) as

$$
\frac{d \hat{a}}{d t}=-\frac{\kappa}{2} \hat{a}+\lambda \hat{m}_{a}
$$

in which $\lambda$ is a constant whose value remains to be fixed. Applying Eq. (22), we get

$$
\left[\hat{a}, \hat{a}^{\dagger}\right]_{k}=\frac{4 g^{2}}{\kappa^{2}}\left(\hat{\eta}_{b}^{k}-\hat{\eta}_{a}^{k}\right)
$$

and on summing over all atoms, we have

$$
\left[\hat{a}, \hat{a}^{\dagger}\right]=\frac{4 g^{2}}{\kappa^{2}}\left(\hat{N}_{b}-\hat{N}_{a}\right)
$$

where

$$
\left[\hat{a}, \hat{a}^{\dagger}\right]=\sum_{k=1}^{N}\left[\hat{a}, \hat{a}^{\dagger}\right]_{k}
$$

stands for the commutator of $\hat{a}$ and $\hat{a}^{\dagger}$ when light mode $a$ is interecting with all the $\mathrm{N}$ three-level atoms. On the other hand, applying the large-time approximation to Eq. (63), one can easily verify that

$$
\left[\hat{a}, \hat{a}^{\dagger}\right]=N \frac{4 \lambda^{2}}{\kappa^{2}}\left(\hat{N}_{b}-\hat{N}_{a}\right) .
$$

Thus on account of Eqs. (65) and (67), we see that

$$
\lambda= \pm \frac{g}{\sqrt{N}} .
$$

In view of Eq. (68), Eq. (63) can be written as

$$
\frac{d \hat{a}}{d t}=-\frac{\kappa}{2} \hat{a}+\frac{g}{\sqrt{N}} \hat{m}_{a}
$$

Following a similar procedure, one can also readily establish that

$$
\begin{gathered}
{\left[\hat{b}, \hat{b}^{\dagger}\right]=\frac{4 g^{2}}{\kappa^{2}}\left(\hat{N}_{c}-\hat{N}_{b}\right),} \\
\frac{d \hat{b}}{d t}=-\frac{\kappa}{2} \hat{b}+\frac{g}{\sqrt{N}} \hat{m}_{b} .
\end{gathered}
$$

Furthermore, in order to include the effect of pumping process, we rewrite Eqs. (33) and (34) as

$$
\begin{aligned}
\frac{d}{d t} \hat{m}_{a} & =-\frac{\mu}{2} \hat{m}_{a}+\hat{G}_{a}(t), \\
\frac{d}{d t} \hat{m}_{b} & =-\frac{\mu}{2} \hat{m}_{b}+\hat{G}_{b}(t)
\end{aligned}
$$

in which $\hat{G}_{a}(t)$ and $\hat{G}_{b}(t)$ are noise operators with vanishing mean and $\mu$ is a parameter whose value remains to be determined. Employing the relation

$$
\frac{d}{d t}\left\langle\hat{m}_{a}^{\dagger} \hat{m}_{a}\right\rangle=\left\langle\frac{d \hat{m}_{a}^{\dagger}}{d t} \hat{m}_{a}\right\rangle+\left\langle\hat{m}_{a}^{\dagger} \frac{d \hat{m}_{a}}{d t}\right\rangle
$$

along with Eq. (72), we easily find

$$
\frac{d}{d t}\left\langle\hat{m}_{a}^{\dagger} \hat{m}_{a}\right\rangle=-\mu\left\langle\hat{m}_{a}^{\dagger} \hat{m}_{a}\right\rangle+\left\langle\hat{m}_{a}^{\dagger} \hat{G}_{a}(t)\right\rangle+\left\langle\hat{G}_{a}^{\dagger}(t) \hat{m}_{a}\right\rangle,
$$


from which follows

$$
\frac{d}{d t}\left\langle\hat{N}_{a}\right\rangle=-\mu\left\langle\hat{N}_{a}\right\rangle+\frac{1}{N}\left[\left\langle\hat{m}_{a}^{\dagger} \hat{G}_{a}(t)\right\rangle+\left\langle\hat{G}_{a}^{\dagger}(t) \hat{m}_{a}\right\rangle\right] .
$$

Hence comparison of Eqs. (59) and (76) shows that

$$
\mu=\gamma+\gamma_{c}+2 r_{a}
$$

and

$$
\left\langle\hat{m}_{a}^{\dagger} \hat{G}_{a}(t)\right\rangle+\left\langle\hat{G}_{a}^{\dagger}(t) \hat{m}_{a}\right\rangle=r_{a} N^{2}
$$

We observe that Eq. (78) is equivalent to

$$
\left\langle\hat{G}_{a}^{\dagger}(t) \hat{G}_{a}\left(t^{\prime}\right)\right\rangle=r_{a} N^{2} \delta\left(t-t^{\prime}\right)
$$

One can also easily verify that

$$
\left\langle\hat{G}_{a}(t) \hat{G}_{a}^{\dagger}\left(t^{\prime}\right)\right\rangle=\left(\gamma+\gamma_{c}\right) N^{2} \delta\left(t-t^{\prime}\right)
$$

Furthermore, adding Eqs. (33) and (34), we have

$$
\frac{d}{d t}\langle\hat{m}\rangle=-\frac{1}{2}\left[\gamma+\gamma_{c}\right]\langle\hat{m}\rangle-\frac{1}{2}\left[\gamma+\gamma_{c}\right]\left\langle\hat{m}_{a}\right\rangle,
$$

where $\hat{m}$ is given by Eq. (53). Upon casting Eq. (81) into the form

$$
\frac{d}{d t} \hat{m}=-\frac{\mu}{2} \hat{m}-\frac{\mu}{2} \hat{m}_{a}+\hat{G}(t)
$$

one can also easily verify that $\mu$ has the value given by Eq. (77) and

$$
\left\langle\hat{G}^{\dagger}(t) \hat{G}\left(t^{\prime}\right)\right\rangle=r_{a} N^{2} \delta\left(t-t^{\prime}\right) .
$$

On the other hand, assuming the atoms to be initial in the bottom level, the expectation value of the solution of Eq. (72) happens to be

$$
\left\langle\hat{m}_{a}(t)\right\rangle=0
$$

Hence the expectation value of the solution of Eq. (69) turns out to be

$$
\langle\hat{a}(t)\rangle=0
$$

In view of Eqs. (69) and (85), we claim that $\hat{a}(t)$ is a Gaussian variable with zero mean. One can also easily verify that

$$
\langle\hat{b}(t)\rangle=0
$$

Then on account of Eqs. (71) and (86), we realize that $\hat{b}(t)$ is a Gaussian variable with zero mean.

Furthermore adding Eqs. (85) and (86), we obtain

$$
\langle\hat{c}\rangle=0
$$

where

$$
\hat{c}=\hat{a}+\hat{b}
$$

In addition, adding Eqs. (69) and (71), we get

$$
\frac{d \hat{c}}{d t}=-\frac{\kappa}{2} \hat{c}+\frac{g}{\sqrt{N}} \hat{m},
$$

where $\hat{m}$ is given by Eq. (53). In view of Eqs. (87) and (89), we see that $\hat{c}$ is a Gaussian variable with zero mean. 


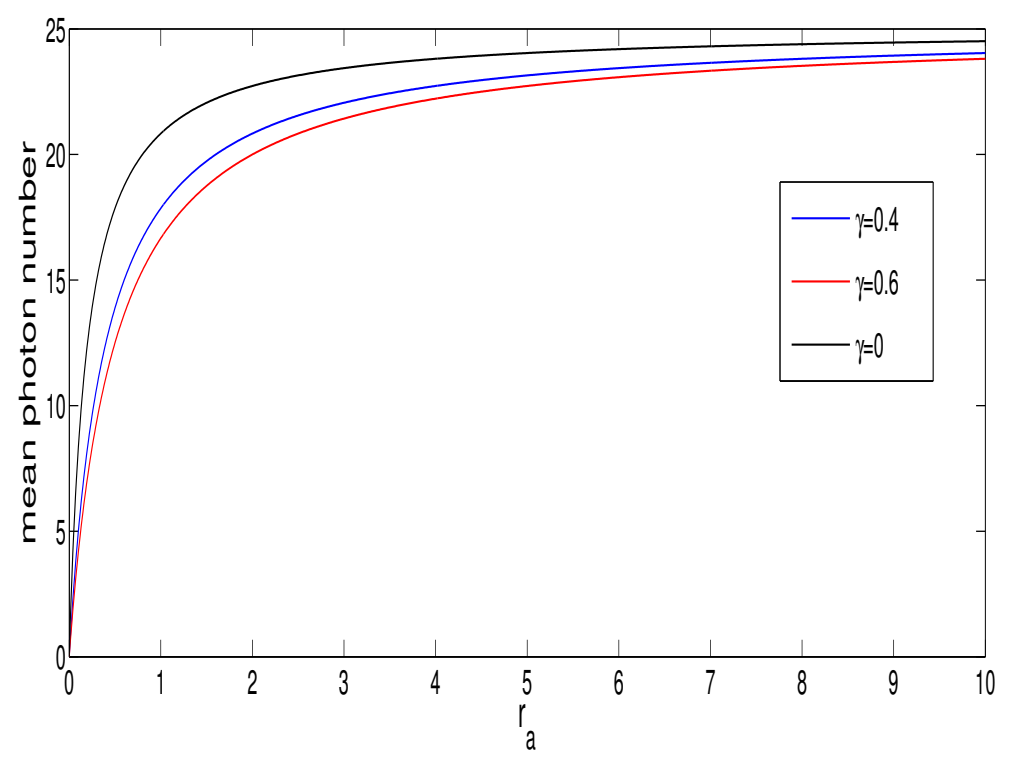

Figure 2. Plots of the mean photon number for the two-mode cavity light at steady state,

[Eq. (94)] for $\kappa=0.8, \gamma_{c}=0.4$, and $N=50$.

\section{Photon statistics}

In this section we wish to calculate the mean and variance of the photon number for the two-mode cavity light at steady state. To this end, the steady-state solution of Eq. (89) has the form

$$
\hat{c}(t)=\frac{2 g}{\kappa \sqrt{N}} \hat{m} .
$$

Thus using Eq. (90) along with Eq. (54), the mean photon number for the two-mode cavity can be written as

$$
\left\langle\hat{c}^{\dagger} \hat{c}\right\rangle=\frac{\gamma_{c}}{\kappa}\left[\left\langle\hat{N}_{a}\right\rangle+\left\langle\hat{N}_{b}\right\rangle\right]
$$

Following a similar procedure, one can establish that

$$
\begin{gathered}
\left\langle\hat{c} \hat{c}^{\dagger}\right\rangle=\frac{\gamma_{c}}{\kappa}\left[\left\langle\hat{N}_{c}\right\rangle+\left\langle\hat{N}_{b}\right\rangle\right], \\
\left\langle\hat{c}^{2}\right\rangle=\frac{\gamma_{c}}{\kappa}\left\langle\hat{m}_{c}\right\rangle .
\end{gathered}
$$

In view of Eqs. (58), (60), and (62), Eqs. (91) and (92) can be rewritten as

$$
\begin{gathered}
\left\langle\hat{c}^{\dagger} \hat{c}\right\rangle=\frac{\gamma_{c}}{\kappa}\left[\frac{2 N r_{a}}{\gamma+\gamma_{c}+2 r_{a}}\right], \\
\left\langle\hat{c} \hat{c}^{\dagger}\right\rangle=\frac{\gamma_{c}}{\kappa}\left[\frac{\gamma+\gamma_{c}+r_{a}}{\gamma+\gamma_{c}+2 r_{a}}\right] N .
\end{gathered}
$$

In the absence of spontaneous emission $(\gamma=0)$, the mean photon number for the two-mode cavity light has the form

$$
\bar{n}=\frac{\gamma_{c}}{\kappa}\left(\frac{2 N r_{a}}{\gamma_{c}+2 r_{a}}\right) .
$$

This result is the same as the one obtained by Fesseha [10]. It can be seen from the plots in Fig. 2 that the presence of spontaneous emission leads to a decrease in the mean photon number for the two-mode cavity light.

Furthermore, the variance of the photon number for the two-mode cavity light is expressible as

$$
(\Delta n)^{2}=\left\langle\left(\hat{c}^{\dagger} \hat{c}\right)^{2}\right\rangle-\left\langle\hat{c}^{\dagger} \hat{c}\right\rangle^{2} \text {. }
$$


Using the fact that $\hat{c}$ is a Gaussian variable with zero mean, we readily get

$$
(\Delta n)^{2}=\left\langle\hat{c}^{\dagger} \hat{c}\right\rangle\left\langle\hat{c} \hat{c}^{\dagger}\right\rangle+\left\langle\hat{c}^{2 \dagger}\right\rangle\left\langle\hat{c}^{2}\right\rangle
$$

We now proceed to calculate the expectation value of the atomic operator $\hat{m}_{c}$ following the approach presented in [10]. To this end, applying the identity given by Eq. (43), the state vector of a three-level atom can be put in the form

$$
\left|\psi_{k}\right\rangle=c_{a}\left|a_{k}\right\rangle+c_{b}\left|b_{k}\right\rangle+c_{c}\left|c_{k}\right\rangle
$$

in which

$$
\begin{aligned}
& c_{a}=\left\langle a_{k} \mid \psi_{k}\right\rangle, \\
& c_{b}=\left\langle b_{k} \mid \psi_{k}\right\rangle, \\
& c_{c}=\left\langle c_{k} \mid \psi_{k}\right\rangle .
\end{aligned}
$$

The state vector described by Eq. (99) can be used to determine the expectation value of an atomic operator formed by a pair of identical energy levels or by two distinct energy levels between which transition with the emission of a photon is dipole forbidden. One can thus readily establish that

$$
\begin{aligned}
& \left\langle\hat{\eta}_{a}^{k}\right\rangle=c_{a} c_{a}^{*}, \\
& \left\langle\hat{\eta}_{c}^{k}\right\rangle=c_{c} c_{c}^{*},
\end{aligned}
$$

and

$$
\left\langle\hat{\sigma}_{c}^{k}\right\rangle=c_{a} c_{c}^{*}
$$

We then see that

$$
\left|\left\langle\hat{\sigma}_{c}^{k}\right\rangle\right|^{2}=\left\langle\hat{\eta}_{a}^{k}\right\rangle\left\langle\hat{\eta}_{c}^{k}\right\rangle
$$

and on taking $\left|\left\langle\hat{\sigma}_{c}^{k}\right\rangle\right|$ to be real, we see that

$$
\left|\left\langle\hat{\sigma}_{c}^{k}\right\rangle\right|=\sqrt{\left\langle\hat{\eta}_{a}^{k}\right\rangle\left\langle\hat{\eta}_{c}^{k}\right\rangle}
$$

so that upon summing over $\mathrm{k}$ from 1 up to $\mathrm{N}$, we get

$$
\left\langle\hat{m}_{c}\right\rangle=\sqrt{\left\langle\hat{N}_{a}\right\rangle\left\langle\hat{N}_{c}\right\rangle} .
$$

On account of this, Eq. (93) takes the form

$$
\left\langle\hat{c}^{2}\right\rangle=\frac{\gamma_{c}}{\kappa} \sqrt{\left\langle\hat{N}_{a}\right\rangle\left\langle\hat{N}_{c}\right\rangle}
$$

Now using Eq. (61), we have

$$
\left\langle\hat{c}^{2}\right\rangle=\frac{\gamma_{c}}{\kappa} \sqrt{\frac{\gamma+\gamma_{c}}{r_{a}}}\left\langle\hat{N}_{a}\right\rangle
$$

In view of Eqs. (91), (92), and (110), Eq. (98) becomes

$$
(\Delta n)^{2}=\frac{\gamma_{c}}{\kappa}\left(\left\langle\hat{N}_{a}\right\rangle+\left\langle\hat{N}_{b}\right\rangle\right)\left(\frac{\gamma_{c}}{\kappa}\left\langle\hat{N}_{b}\right\rangle+\left\langle\hat{N}_{c}\right\rangle\right)+\left(\frac{\gamma_{c}}{\kappa} \sqrt{\frac{\gamma+\gamma_{c}}{r_{a}}}\left\langle\hat{N}_{a}\right\rangle\right)^{2} .
$$

Finally, on account of Eqs. (58), (60), (61), and (62) along with Eq. (111), we arrive at

$$
(\Delta n)^{2}=\frac{1}{4} \bar{n}^{2}(3 \eta+2),
$$

where

$$
\eta=\frac{\gamma+\gamma_{c}}{r_{a}}
$$

Now, inspection of Eq. (112) indicates that $(\Delta n)^{2}>\bar{n}$ and hence the photon statistics of the two-mode cavity light is superPoissonian. 


\section{Quadrature squeezing}

We now proceed to calculate the quadrature squeezing of the two-mode cavity light in the entire frequency interval. To this end, the squeezing properties of the two-mode cavity light are described by two quadrature operators defined by

$$
\hat{c}_{+}=\hat{c}^{\dagger}+\hat{c}
$$

and

$$
\hat{c}_{-}=i\left(\hat{c}^{\dagger}-\hat{c}\right)
$$

It can be readily established that [14]

$$
\left[\hat{c}_{-}, \hat{c}_{+}\right]=2 i \frac{\gamma_{c}}{\kappa}\left(\left\langle\hat{N}_{a}\right\rangle-\left\langle\hat{N}_{c}\right\rangle\right)
$$

It then follows that

$$
\Delta c_{+} \Delta c_{-} \geq \frac{\gamma_{c}}{\kappa}\left|\left\langle\hat{N}_{a}\right\rangle-\left\langle\hat{N}_{c}\right\rangle\right|
$$

Upon setting $r_{a}=0$, we see that

$$
\Delta c_{+} \Delta c_{-} \geq \frac{\gamma_{c}}{\kappa} N
$$

The variance of the quadrature operator is expressible as

$$
\left(\Delta c_{ \pm}\right)^{2}= \pm\left\langle\left(\hat{c}^{\dagger} \pm \hat{c}\right)^{2}\right\rangle \mp\left[\left\langle\hat{c}^{\dagger}+\hat{c}\right\rangle\right]^{2},
$$

so that on account of Eq. (87), we have

$$
\left(\Delta c_{ \pm}\right)^{2}=\left\langle\hat{c}^{\dagger} \hat{c}\right\rangle+\left\langle\hat{c} \hat{c}^{\dagger}\right\rangle \pm\left\langle\hat{c}^{\dagger 2}\right\rangle \pm\left\langle\hat{c}^{2}\right\rangle .
$$

Now employing Eqs. (44), (91), (92), and (110), we arrive at

$$
\begin{aligned}
& \left(\Delta c_{+}\right)^{2}=\frac{\gamma_{c}}{\kappa}\left(N+\left\langle\hat{N}_{a}\right\rangle+2 \sqrt{\frac{\gamma+\gamma_{c}}{r_{a}}}\left\langle\hat{N}_{a}\right\rangle\right), \\
& \left(\Delta c_{-}\right)^{2}=\frac{\gamma_{c}}{\kappa}\left(N+\left\langle\hat{N}_{a}\right\rangle-2 \sqrt{\frac{\gamma+\gamma_{c}}{r_{a}}}\left\langle\hat{N}_{a}\right\rangle\right) .
\end{aligned}
$$

Moreover, on setting $r_{a}=0$ in Eqs. (121) and (122), we get

$$
\left(\Delta c_{+}\right)_{v}^{2}=\left(\Delta c_{-}\right)_{v}^{2}=\frac{\gamma_{c}}{\kappa} N .
$$

This represents the quadrature variance of a two-mode cavity vacuum state. From Eqs. (118) and (123), we see that the two-mode cavity light is in a minimum uncertainty state. We seek to calculate the quadrature squeezing of the two-mode cavity light relative to the quadrature variance of the two-mode cavity vacuum state. We then define the quadrature squeezing of the two-mode cavity light by

$$
S=\frac{\left(\Delta c_{-}\right)_{v}^{2}-\left(\Delta c_{-}\right)^{2}}{\left(\Delta c_{-}\right)_{v}^{2}} .
$$

Now employing Eqs. (122) and (123), one can put Eq. (124) in the form

$$
S=\frac{\frac{\gamma_{c}}{\kappa}\left(2 \sqrt{\frac{\gamma+\gamma_{c}}{r_{a}}}\left\langle\hat{N}_{a}\right\rangle-\left\langle\hat{N}_{a}\right\rangle\right)}{\frac{\gamma_{c}}{\kappa} N} .
$$

On account of Eq. (60), Eq. (125) takes the form

$$
S=\left(\frac{2 \sqrt{\frac{\gamma+\gamma_{c}}{r_{a}}}-1}{\frac{\gamma+\gamma_{c}}{r_{a}}+2}\right) .
$$

We note that, unlike the mean photon number, the quadrature squeezing does not depend on the number of atoms. This implies that the quadrature squeezing of the cavity light is independent of the number of photons. The plots in Fig. 3 indicate that the maximum quadrature squeezing is $50 \%$ both for $\gamma=0, \gamma=0.4$, and $\gamma=0.6$. This occurs when the three-level laser is operating at $r_{a}=0.1, r_{a}=0.2$, and $r_{a}=0.3$, respectively. This result is the same as the one obtained by Fesseha [10]. 


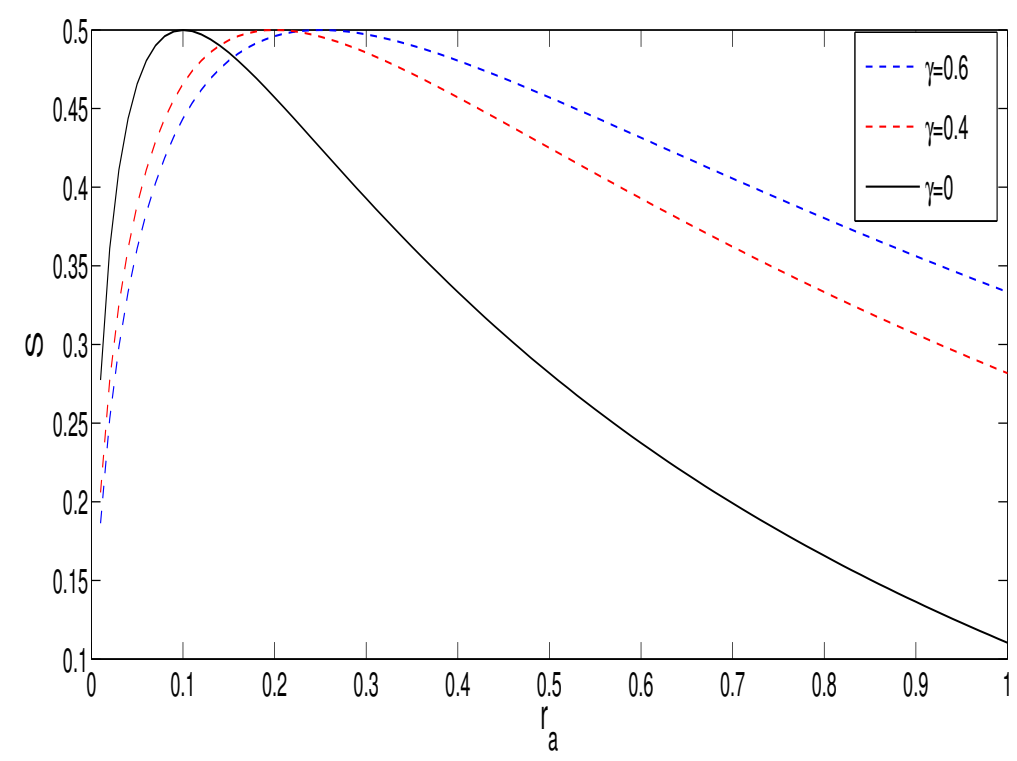

Figure 3. Plots of the quadrature squeezing at steady state,

[Eq. (126)] versus $r_{a}$ for $\kappa=0.8, \gamma_{c}=0.4$

\section{Conclusions}

In this paper we have studied the statistical and squeezing properties of the light generated by three-level atoms available in an open cavity and pumped to the top level by electron bombardment at constant rate. We have carried out our analysis by putting the noise operators associated with the vacuum reservoir in normal order. Applying the large-time approximation scheme, we have obtained the steady-state solutions of the equations of evolution for the expectation values of the atomic operators and the quantum Langevin equations for the cavity mode operators.

Using the resulting steady-state solutions, we have calculated the mean photon number, the variance of the photon number, and the quadrature variance for the two-mode cavity light. We have seen that the light generated by the three-level laser is in a squeezed state and the squeezing occurs in the minus quadrature. It so turns out that the maximum quadrature squeezing of the two-mode cavity light is $50 \%$ for $\gamma=0, \gamma=0.4$, and $\gamma=0.6$ below the vacuum-state level. Our result shows that the maximum quadrature squeezing is the same as the one obtained by Fesseha [10].

\section{REFERENCES}

[1] M.O. Scully, K. Wodkiewicz, M.S. Zubairy, J. Bergou, N. Lu, J. Meyer ter Vehn, Phys.Rev. Lett. 60, 1832 (1988).

[2] Fesseha Kassahun, Fundamentals of Quantum Optics (Lulu Press Inc., North Carolina, 2010).

[3] N. Lu, F.X. Zhao, J. Bergou, Phys. Rev. A 39, 5189 (1989.

[4] N. A. Ansari, Phys. Rev. A 48, 4686 (1993).

[5] Tewodros Y. Darge and Fesseha Kassahun, PMC Physics B, 1 (2010).

[6] E. Alebachew and K. Fesseha, Opt. Commun. 265, 314 (2006).

[7] Tamirat Abebe,Coherently driven Nondegenerate Three-Level Laser with Noiseless Vacuum Reservoir, Bulg. J. Phys. 30 (2005).

[8] N.A. Ansari, J. Gea-Banacloche, and M.S. Zubairy, Phys. Rev. A 41, 5179 (1990).

[9] Fesseha Kassahun, ArXiv:1105.1438v3 [quant-ph] 25 Sep 2012.

[10] Fesseha Kassahun, Refined Quantum Analysis of Light, Revised Edition (CreateSpace Independent Publishing Platform, 2016).

[11] C.A. Blockely and D.F. Walls, Phys. Rev. A 43, 5049 (1991).

[12] K. Fesseha, Phys. Rev. A63, 033811 (2001). 
[13] Fesseha, K. Stimulated Emission by Two-Level Atoms Pumped to the Upper Level. Optics Communications, 284, 1357-1363 (2011).

[14] Fesseha Kassahun, The commutation relation for cavity mode operators, ArXiv.1611.01003v2[quan-Ph] 29 March 2018. 